\title{
Characterization of the genome and transcriptome of the blue tit Cyanistes caeruleus: polymorphisms, sex-biased expression and selection signals
}

\author{
JAKOB C. MUELLER,${ }^{* 1}$ HEINER KUHL,$\dagger^{1}$ BERND TIMMERMANN† and BART KEMPENAERS* \\ *Department of Behavioural Ecology \& Evolutionary Genetics, Max Planck Institute for Ornithology, 82319 Seewiesen, Germany, \\ $\dagger$ Sequencing Core Facility, Max Planck Institute for Molecular Genetics, Ihnestrasse 73, 14195 Berlin, Germany
}

\begin{abstract}
Decoding genomic sequences and determining their variation within populations has potential to reveal adaptive processes and unravel the genetic basis of ecologically relevant trait variation within a species. The blue tit $C y a n i s t e s$ caeruleus - a long-time ecological model species - has been used to investigate fitness consequences of variation in mating and reproductive behaviour. However, very little is known about the underlying genetic changes due to natural and sexual selection in the genome of this songbird. As a step to bridge this gap, we assembled the first draft genome of a single blue tit, mapped the transcriptome of five females and five males to this reference, identified genomewide variants and performed sex-differential expression analysis in the gonads, brain and other tissues. In the gonads, we found a high number of sex-biased genes, and of those, a similar proportion were sex-limited (genes only expressed in one sex) in males and females. However, in the brain, the proportion of female-limited genes within the female-biased gene category $(82 \%)$ was substantially higher than the proportion of male-limited genes within the male-biased category (6\%). This suggests a predominant on-off switching mechanism for the female-limited genes. In addition, most male-biased genes were located on the Z-chromosome, indicating incomplete dosage compensation for the male-biased genes. We called more than 500000 SNPs from the RNA-seq data. Heterozygote detection in the single reference individual was highly congruent between DNA-seq and RNA-seq calling. Using information from these polymorphisms, we identified potential selection signals in the genome. We list candidate genes which can be used for further sequencing and detailed selection studies, including genes potentially related to meiotic drive evolution. A public genome browser of the blue tit with the described information is available at http://public-genomes-ngs.molgen.mpg.de.
\end{abstract}

Keywords: blue tit, brain/gonad transcriptome, Cyanistes caeruleus, de novo genome, meiotic drive, RNA-seq, sex-biased expression, signatures of selection, SNP calling

Received 7 April 2015; revision received 22 July 2015; accepted 22 July 2015

\section{Introduction}

A major goal in evolutionary ecology is to identify and understand adaptive processes from the perspective of genes, individuals and populations with a focus on the individual phenotypic and genomic level (Springer et al. 2011; Diz et al. 2012). Whereas the phenotypic approach can be used to analyse and detect ongoing processes of adaptation and potential constraints on adaptation by pleiotropy and plasticity, the genomic approach has the potential to reveal adaptive processes over a longer time-

Correspondence: Jakob C. Mueller, Fax: ++49-8157-932-400; E-mail: mueller@orn.mpg.de

${ }^{1}$ Shared first authorship. scale including past selective episodes in the population history (Van Oers \& Mueller 2010). The combined investigation of the maintenance and dynamics of phenotypic and genomic variation in populations is important for understanding the potential for trait evolution. However, for many species, the knowledge about genomic evolution substantially lags behind the knowledge about phenotypic evolution.

The blue tit (Cyanistes caeruleus) and the closely related great tit (Parus major) have been established as ecological model species for more than 60 years (Kluyver 1951; Lack 1964). Since then, fitness consequences of variation in many life history and behavioural traits have been described in the wild (Merilä \& Sheldon 1999; Ellegren \& Sheldon 2008; Clutton-Brock \& Sheldon 
2010). In blue tits, a specific focus has been on extra-pair mating behaviour (for recent publications see Vedder et al. 2011; Schlicht \& Kempenaers 2013; Arct et al. 2013). However, only a few studies of genotype-phenotype or genotype-fitness relationships have been performed (Olano-Marin et al. 2011a,b; Kluen et al. 2012 and Steinmeyer et al. 2012). This is mainly due to the paucity of genomic information in this species; so far, only random genomic markers - although large sets - have been developed (e.g. Olano-Marin et al. 2010). Genome and transcriptome sequencing will help to bridge this gap.

High-throughput (next-generation) sequencing techniques enable the generation of new avian genome databases (Zhang et al. 2014), which can then be improved with the help of 'reference' genome assemblies such as that of the chicken (Gallus gallus) and zebra finch (Taeniopygia guttata). These reference genomes have a chromosomal scale and have been assembled with the help of genetic linkage maps (ICGSC 2004; Warren et al. 2010). Reference species facilitate the assembly of new bird genomes, particularly because chromosomal organization appears to be highly conserved among avian taxa (Ellegren 2010; Frankl-Vilches et al. 2015). Recently, bird genomes that have attracted particular interest are those of species with clear categories of domesticated breeds (Dalloul et al. 2010), morphotypes (Shapiro et al. 2013) or subspecies (Ellegren et al. 2012; Poelstra et al. 2014), or that are adapted to extreme environments or lifestyles (Cai et al. 2013; Huang et al. 2013; Zhan et al. 2013). However, the publication of genome information on ecological model species should be a helpful longlasting resource for micro-evolutionary studies in the field.

The general aim of this study was to provide a detailed description of the blue tit genome, transcriptome and genetic polymorphisms in order to promote genetic studies on ecologically relevant traits in the field. Here, we report on (i) the assembly of the blue tit genome, assisted by the Tibetan ground tit (Pseudopodoces/Parus humilis) and chicken genome, and (ii) the characterization of the blue tit transcriptome, based on sequencing of different tissue samples of five males and five females. We analysed gene expression in the female and male gonads and brain in comparison with the pooled remaining tissues to (iii) describe general patterns of sex-differential gene expression in brain and gonads and to (iv) list potential candidate genes for sexual conflict and/or rapid evolution (Parsch \& Ellegren 2013). After identifying genomic variants in the transcribed regions, we used their population frequencies, patterns of linkage disequilibrium and functional annotation to (v) reveal potential loci under selection.

\section{Materials and methods}

\section{Sampling}

We collected 10 adult blue tits (five males and five females) under licence (Regierung von Oberbayern, Az. 55.1-8642.3-45-2011) from a wild population in a natural forest reserve in southern Germany (Westerholz, $48^{\circ} 08^{\prime} \mathrm{N}, 10^{\circ} 53^{\prime} \mathrm{E}$; for details of the area see Steinmeyer et al. 2012). We caught all individuals inside a nestbox at the beginning of the breeding season, between 28 March and 3 April 2012, while they were inspecting a box or roosting inside. Individuals were brought to the laboratory, sacrificed and directly dissected to extract brain, gonads, skin, pectorals, heart, lung, liver, gizzard, spleen, pancreas, intestine and kidney. Halves of each organ were directly frozen or stabilized in RNAlater (Qiagen). A blood sample of one of the male birds (reference bird BT333_1) was stored in Queen's lysis buffer (Seutin et al. 1991) for DNA extraction.

\section{DNA/RNA extraction and sequencing}

DNA was extracted from the blood sample with the Nucleospin Blood QuickPure kit (Macherey-Nagel) and RNA was extracted from the organ samples with RNeasy Mini kits (Qiagen) according to the manufacturer's recommendations. In each sample, we measured RNA concentration with a Bioanalyzer (Agilent Technologies). RNA samples were pooled into three tissue groups per individual for sequencing: total brain, gonads and all other remaining organs (equal RNA amounts).

The genome of the single male reference blue tit (BT333_1) was sequenced by the Illumina HiSeq 2000 technique using a 500-bp and a 1000-bp insert pairedend library with 101-bp read length. The transcriptome samples of the 10 blue tit individuals (three tissue samples per individual) were separately prepared and filtered for RNAs with poly-A tails using Illumina's TruSeq RNA Sample Preparation Kits before sequencing by the Illumina HiSeq paired-end technique with read lengths of 50 or $101 \mathrm{bp}$ (for the reference individual BT333_1) and an insert size of about $200 \mathrm{bp}$. For sequencing details including barcodes for each sample and SRA accession numbers see Table S1 (Supporting information).

\section{Genome assembly and gene annotation}

We filtered and trimmed the whole genome shotgun raw sequences of blue tit BT333_1 to obtain high-quality nonduplicate read pairs. This was achieved by a custom script that extracted only the largest parts of reads that were longer than $32 \mathrm{bp}$ and contained no base with 
Phred quality score lower than 11 (Frankl-Vilches et al. 2015). We compared the first $32 \mathrm{bp}$ of each read in a pair to those of other read pairs and kept only one pair if the same sequence was found more than once (deduplication). The reads were assembled by a hybrid strategy which involved (i) the assembly of all short reads by a 'de Bruijn graph' assembler using IDBA-UD v1.1.1 (Peng et al. 2012), and (ii) assembling the resulting contigs and a subset of the short read data by a 'overlap layout consensus' long read assembler using CELERA ASSEMBLER v7 (Myers et al. 2000). For more details regarding parameters applied see Appendix S1 (Supporting information). Some putative interchromosomal missassemblies were identified by whole genome alignment using LAST v266 (Kielbasa et al. 2011; Appendix S1, Supporting information) with the Tibetan ground tit (Pseudopodoces/Parus humilis; Cai et al. 2013; GenBank Accession no. ANZD00000000). First, the Tibetan ground tit scaffold identifiers were assigned to chicken chromosomes by whole genome alignment. The so identified missassemblies were removed by splitting the corresponding blue tit scaffolds. Superscaffolds were subsequently established by colinearity with the Tibetan ground tit genome as described in Frankl-Vilches et al. (2015). The final blue tit assembly was compared with the chicken assembly using LAST to assign putative chromosome IDs to the superscaffolds. Additionally, we assigned the blue tit superscaffolds to zebra finch chromosomes (Table S2, Supporting information) in order to take into account the characteristic chromosome splits in the passerine lineage (e.g. on chromosomes 1 and 4).

Protein-coding sequences in the blue tit genome were annotated by aligning all avian protein sequences of the NCBI and Ensembl protein database (reference date: 19 May 2014) using SPALN v2.1.2 (Gotoh 2008; Appendix S1, Supporting information; see track 'Aligned Proteins' in the genome browser). A single best protein match for each cluster of gene predictions in the genome was selected according to the best SPALN alignment score (which summarizes alignment length and identity of a match, thus preferring more complete predictions from less diverged species). We used two steps to remove redundant transcripts that had lower SPALN scores based on genomic location. First, we removed transcripts with strand-specific exact overlap of single exons between two or more transcript models. Second, we removed residual redundant transcripts that had strandspecific, but not exact, overlaps (see track 'Best Protein Match' in the genome browser). Gene names were assigned by these alignments. We further refined the coding gene annotation - in particular the UTR annotation - by merging SPALN predictions (which had been converted to gtf format) with the RNA-seq/Cufflinksderived transcript models of the male BT333_1 using
Cuffmerge (Trapnell et al. 2010). As coding sequence annotation is lost during this step, we used TransDecoder r20131117 (https://transdecoder.github.io/) to reassign ORF information for protein translation (see 'Protein Coding Genes' track in the genome browser).

\section{Transcriptome mapping and assembly}

The transcriptome sequence reads (RNA-seq) of each tissue sample from each individual were mapped to the genome sequence using торнат2 v2.0.9 (Kim et al. 2011; Appendix S1, Supporting information), with support of a splice-junction database from earlier versions of genome annotations (we used predicted exon/intron boundaries from the SPALN protein alignments as well as exon/intron boundaries from the first round of TOPнAт using the 101-bp RNA-seq reads from BT333_1). Parsimonious sets of transcripts for each tissue type were assembled using CUFFLINKS v2.1.1 (Trapnell et al. 2010; Appendix S1, Supporting information). This step provides a complete picture of the transcriptome, because it takes the untranslated regions of genes into account, and considers differential splicing and poly-adenylated long noncoding RNA and miRNA precursors (Guttman et al. 2009; Necsulea \& Kaessmann 2014; Philippe et al. 2014). The Cufflinks-predicted exons mapped to the genome were first merged across the three tissues within each individual using Cuffmerge and then intersected across groups of individuals using BEDTOOLs v2.19.1 (Quinlan \& Hall 2010) to reveal the following four groups: (i) exons common to all males for the comparison of RNA-seq and DNA-seq variants in the single reference male, (ii) exons common to all individuals, (iii) exons common to all females (but not found in all males) and (iv) exons common to all males (but not found in all females). According to the three last groups, we categorized SNPs identified from the transcriptome as common to both sexes or as detected exclusively in the female- or male-specific transcriptome.

\section{Sex-specific expression analysis}

We tested for sex-differential expression of the 67359 Cufflinks-predicted genes in the three tissue samples using Cuffdiff (Trapnell et al. 2013; Appendix S1, Supporting information). This algorithm estimates expression as FPKM (fragments per kilobase of transcript per million mapped reads) at transcript/isoform-level resolution and controls for variability across replicates (five males and five females). We allowed a false discovery rate of 0.05 . Among the genes that showed sex-biased expression, we discriminated between those that were expressed in both sexes and those that were only expressed in either males or females (sex-limited 
expression; FPKM $<=0.0001$ in one sex). The proportion of annotated genes in the sex-biased and unbiased category was estimated by the relative number of Cufflinks-predicted genes, which showed positional overlap with best protein hit regions, using BEDTOOLS.

\section{Variant calling and annotation}

After removing duplicate reads (using PICARD v1.122, Appendix S1, Supporting information), we called SNPs and indels (insertions/deletions) and assigned individual genotypes from both the single-individual DNA-seq data and the 10-individual RNA-seq data using the HaplotypeCaller of GATK v3.2 (DePristo et al. 2011; Appendix S1, Supporting information). The genotypes of the RNA-seq data were determined for each of the 10 individuals in a single step following GATK best practices (Van der Auwera et al. 2013; Appendix S1, Supporting information). The variant sets of both the DNA-seq and RNA-seq data were then filtered according to fixed rules from the GATK best practices recommendations (Van der Auwera et al. 2013; Appendix S1, Supporting information) and to coverage (included if read depth per individual $>6$ ). We also excluded high-coverage variants (read depth $>99$ th percentile of base coverage) from the genomic set to avoid variant calls from duplicated genome regions. To filter out potential false-positive calls due to mapping errors, we allowed a maximal indel length of $10 \mathrm{bp}$.

Variants were functionally annotated according to the gene position using SNPEFF v3.5 (Cingolani et al. 2012). Here, gene position was defined by the refined protein annotation ('Protein Coding Genes' track in genome browser).

\section{Scan for selection signatures}

We applied three different methods for the detection of potential selection signals in the RNA-seq SNP data. Our focus was on methods which are able to detect ongoing selection in small genomic regions, but also included a standard selection scan of allele frequency spectra. Only SNPs that were scored in all 10 individuals were used, because allele and haplotype frequencies need to be estimated, and SNPs located in the homologous region of chromosome $\mathrm{Z}$ were excluded due to its hemizygotic nature in females. In all tests, we applied a window size of $25 \mathrm{~kb}$, which is close to the average size of avian genes (see e.g. chicken genome at genome.ucsc.edu). The genes identified as candidates for selection were tested for functional enrichment in gene ontology (GO) categories using g:Profiler (Reimand et al. 2011) with the 11827 annotated G. gallus Ensemble genes as background/null distribution (Ensemble genomes 22). $P$-values were corrected for multiple testing by the native method implemented in g:Profiler.

1 Tajima's $D$ based on unphased genotypes was calculated for all adjacent $25-\mathrm{kb}$ windows within each chromosome/scaffold using vCFTOOLS v0.1.11 (Danecek et al. 2011). Clusters of a minimum of two adjacent windows with $|\mathrm{D}|>1.96$ were considered interesting. This test is mostly sensitive to historical selective sweeps and balancing selection.

2 All SNPs were phased and imputed within each chromosome/scaffold based on the physical sequence reads and population frequency information using SHAPEIT v2.790 (Delaneau et al. 2013). This method is based on the Gibbs' sampling scheme in which each individual's haplotypes are sampled conditionally on both the current estimates of all other individuals and the phase-informative sequencing reads of the individual (Delaneau et al. 2013). The latter increases the reliability of the estimated short-distance haplotypes. The estimation of long-distance haplotypes, however, is more error-prone given our small sample of 10 individuals, particularly across regions with no SNPs. After phasing, we calculated the standardized log-ratio of the integrated extended haplotype homozygosities of both alleles at each SNP (iHS according to Voight et al. 2006) using the $\mathrm{R}$ package REHH V1.11 (Gautier \& Vitalis 2012). The iHS test statistic should be mostly independent of local variation in recombination rates, because both the reference and the alternate allele are located at the same genomic position. It is standardized within the frequency classes of the minor allele in 5\% bins between $10 \%$ and $50 \%$. SNPs with minor allele frequency $<10 \%$ were excluded. The iHS statistic has an approximately standard normal distribution and provides a measure of how unusual the haplotype extensions around a given SNP are, relative to the haplotype frequencies. As simulations have shown that selection produces clusters of extreme iHS values (Voight et al. 2006), we considered a minimum of 10 absolute iHS values $>3.09$ $(0.1 \%$ level $)$ within any window of $25 \mathrm{~kb}$ indicative for a partial selective sweep.

3 For the annotated gene regions, we also considered clusters of common nonsynonymous SNPs (NS-SNPs) as interesting; that is, we determined all cases of at least seven NS-SNPs with minor allele frequency $>0.35$ within any $25 \mathrm{~kb}$ window. Such cases might indicate balancing selection maintaining high structural protein variation or ongoing (partial) selective sweeps on haplotypes with strong impact on structural variation.

All these selection scans are restricted to genomic regions transcribed in both sexes. However, the 
detection power might still depend on the local transcriptome coverage and therefore SNP density in these regions. To assess the severity of this potentially confounding factor, we tested the strength of the correlations between Tajima's $D$ or mean absolute iHS values and transcript coverage across all neighbouring $25-\mathrm{kb}$ windows. Transcript coverage and SNP density in these windows were correlated $(r=0.42 ; 95 \% \mathrm{CI}$ : $0.41-0.44)$.

\section{Results}

\section{Genome}

DNA sequencing yielded more than 54 Gb in 543 million high-quality reads. The resulting draft assembly comprised about $1099 \mathrm{Mb}$ in 54108 contigs with an N50 length of $85.7 \mathrm{~kb}$, or - after reference-assisted scaffolding - in 29311 scaffolds with an N50 length of $16.8 \mathrm{Mb}$. Median base coverage of the assembly was $47 \times(5$ th percentile: $15 \times$; 95 th percentile: $68 \times$ ).

Ninety-one per cent of the blue tit assembly (394 superscaffolds) could be mapped to chicken chromosomes or linkage groups. All chromosomes or linkage groups of the assembled chicken genome aligned with the blue tit genome except the smaller chromosome 32 and the sex chromosome W. The latter was not sequenced with our single male blue tit.
We found 21434 best protein gene hits on the genome, which comprised $37 \%$ of the genome size (including introns). Alignment sites of these hits (180 549 protein-coding exons) covered $2.6 \%$ of the genome size (for comparison see e.g. chicken genome at genome. ucsc.edu). Using our transcriptome data, we obtained complete gene annotations including UTRs for 17062 of the coding genes defined above, which increased the annotated genome size to $43 \%$ (6\% in exons).

The public blue tit genome browser can be found at http://public-genomes-ngs.molgen.mpg.de. Figure 1 shows an example screen shot of the browser at the gene end of MKI67. Known protein-coding genes are annotated in the 'Best Protein Match' and 'Aligned Proteins' track and transcribed regions can be inferred from the 'TRANSCRIPTOME' tracks. By combining the information of these tracks, as done in the 'Protein Coding Genes' track, one can identify UTRs. The 'MAF2' track shows the alignments with the ground tit, medium ground finch (Geospiza fortis) and zebra finch. The current chromosome naming refers to chicken chromosomes. Assignments to zebra finch chromosomes can be found in Table S2 (Supporting information). Identified SNPs are visible in the 'SNP' tracks. The browser has the characteristic functionalities of a UCSC browser (genome.ucsc.edu) including BLAT searches, DNA sequence extraction and table browser. Use the help button for further information.

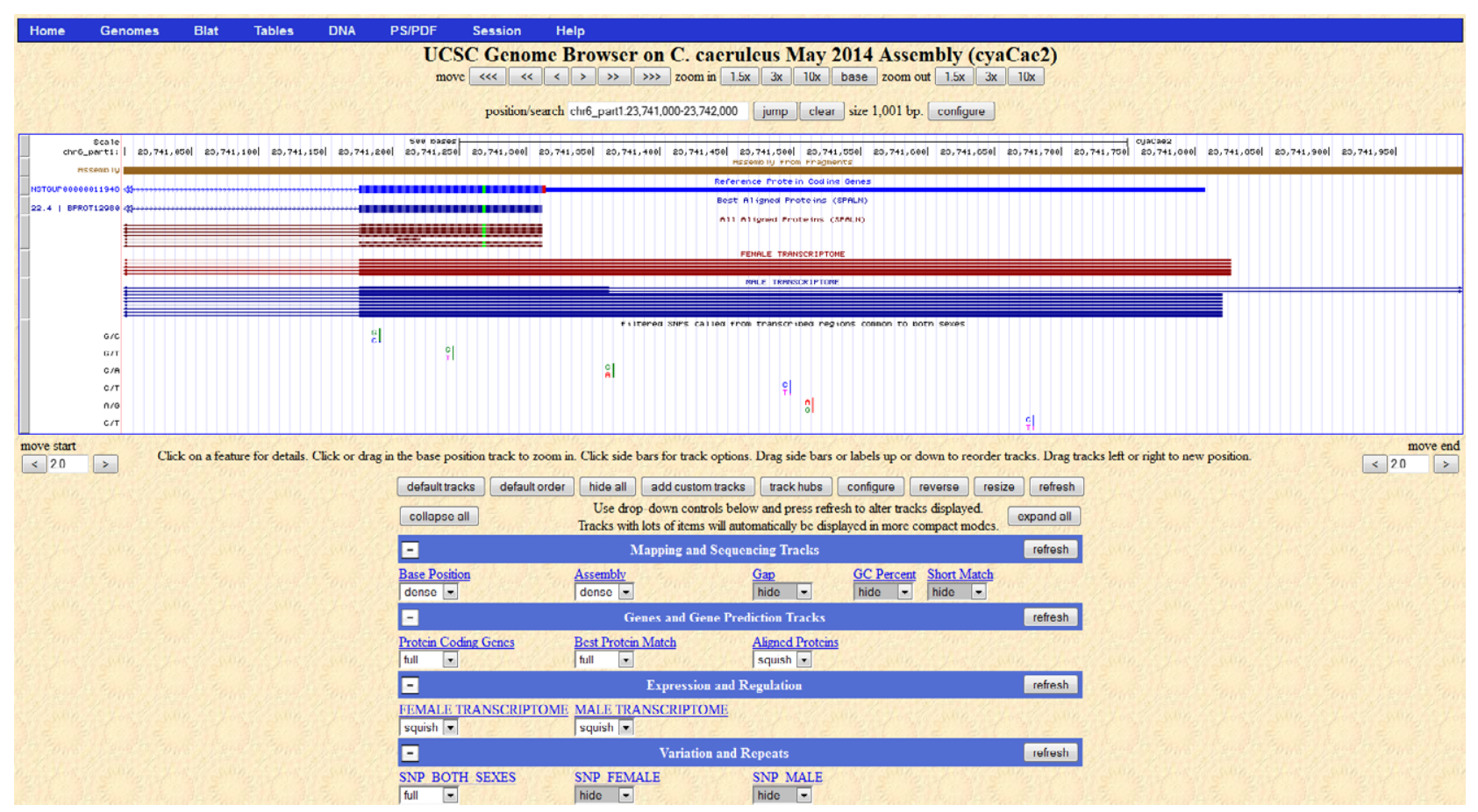

Fig. 1 Example screen shot of the blue tit browser showing the $3^{\prime}$ end of gene MKI67. 


\section{Transcriptome}

RNA sequencing yielded between 91 and 195 million high-quality reads per individual and tissue sample (brain, gonads and pooled other organs). Merging all Cufflinks transcript predictions of all individuals and tissues revealed 67359 predicted genes/transcribed regions (including coding and noncoding 'genes', see Necsulea \& Kaessmann 2014) and 209472 isoforms. Most (82\%) of the best protein hits overlapped one of the Cufflinks-predicted genes. However, only 33\% of the Cufflinks-predicted genes overlapped with best protein hits, leaving a substantial number of transcripts nonannotated and/or nonprotein coding.

In addition, we report the overlapping/intersecting genomic regions of Cufflinks-predicted exons common to both sexes, specific to females and specific to males. This is useful for filtering SNPs for which genotype information is available for all 10 individuals or only for a single sex. A total of 155340 exons $(58.5 \mathrm{Mb})$ were common to all individuals, 18813 exons $(7.5 \mathrm{Mb})$ were specific to females, and 37477 exons $(17.2 \mathrm{Mb})$ were specific to males.

\section{Sex-specific expression}

As expected, a high number of genes were differentially expressed between testis and ovaries: 22389 of 42128 expressed genes showed a significant sex bias (53\%, including sex-limited genes; Table 1; Fig. 2). More genes showed a higher or exclusive expression in males than in females (Table 1). The proportion of Z-chromosomal vs. autosomal location was larger for the male-biased $(8 \%)$ than for the female-biased (4\%) genes $\left(\chi^{2}=176.8\right.$, d.f. $=1, P<0.0001)$. A relatively low proportion of the genes expressed in gonads overlapped best protein hits in both the unbiased (52\%) and sex-biased genes (56\%),

Table 1 Number of differentially expressed genes in the three tissue samples of blue tits. Percentages within the respective higher level group are given in brackets

\begin{tabular}{cccc}
\hline & Gonads & Brain & $\begin{array}{l}\text { Pooled } \\
\text { other tissues }\end{array}$ \\
\hline $\begin{array}{l}\text { Expressed genes } \\
\text { (total) }\end{array}$ & 42128 & 24431 & 22761 \\
Male-biased & $12071(29 \%)$ & $325(1.3 \%)$ & $84(0.4 \%)$ \\
Male-limited & $2183(18 \%)$ & $20(6 \%)$ & $19(23 \%)$ \\
Z-chromosomal & $926(8 \%)$ & $206(63 \%)$ & $8(10 \%)$ \\
Female-biased & $10318(24 \%)$ & $278(1.1 \%)$ & $202(0.9 \%)$ \\
Female-limited & $1025(10 \%)$ & $227(82 \%)$ & $141(70 \%)$ \\
Z-chromosomal & $363(4 \%)$ & $23(8 \%)$ & $13(6 \%)$ \\
\hline
\end{tabular}

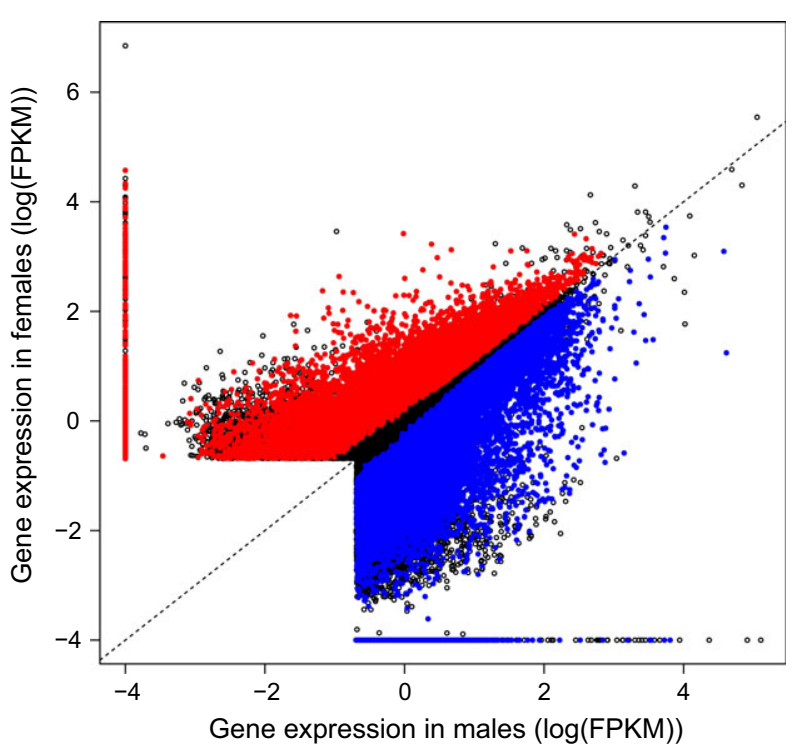

Fig. 2 Sex-specific expression of 42128 genes in the gonads of blue tits. Genes with low numbers of read alignments in both sexes ( $<10$ fragment alignments) were not tested (lower left corner in plot). The lowest expression in a single sex was set to 0.0001 FPKM $[=-4 \log (\mathrm{FPKM})$, fragments per kilobase of transcript per million mapped reads] and indicates genes with sexlimited expression. Significant male-biased expression is shown in blue and significant female-biased expression in red. The dotted line indicates equal expression. Nonsignificance can also be due to large variance in expression between individuals of one sex.

indicating that a high number of genes expressed in the gonads remain nonannotated.

In the brain, we found 603 of 24431 tested genes that were expressed differentially between the sexes (2.5\%; Table 1; Fig. 3). Although there was no difference in the number of genes with male-biased vs. female-biased expression $\left(\chi^{2}=3.55\right.$, d.f. $\left.=1, P=0.059\right)$, the number of genes that were expressed only in females (227) was significantly larger than the number of genes exclusively expressed in males $\left(20 ; \chi^{2}=350.0\right.$, d.f. $\left.=1, P<0.0001\right)$. Thus, most of the genes with male-biased expression belong to the group of genes which are expressed in both sexes (see Fig. 3). Of the 603 genes that showed sex-biased expression in the brain, 229 were found on the Z-chromosome. The majority of these genes (206) showed a male-biased expression (sex difference: $\chi^{2}=344.4$, d.f. $\left.=1, \quad P<0.0001\right)$. Those genes that showed a sex-biased expression were less likely to be annotated with best protein hits (52\%) than the unbiased genes $(71 \%)$. This difference was due to the very low annotation rate among genes with female-biased expression ( $14 \%$ vs. $85 \%$ for male-biased genes).

In the pooled samples from the other organs, as expected, only few genes (286 of 22761 tested genes) 


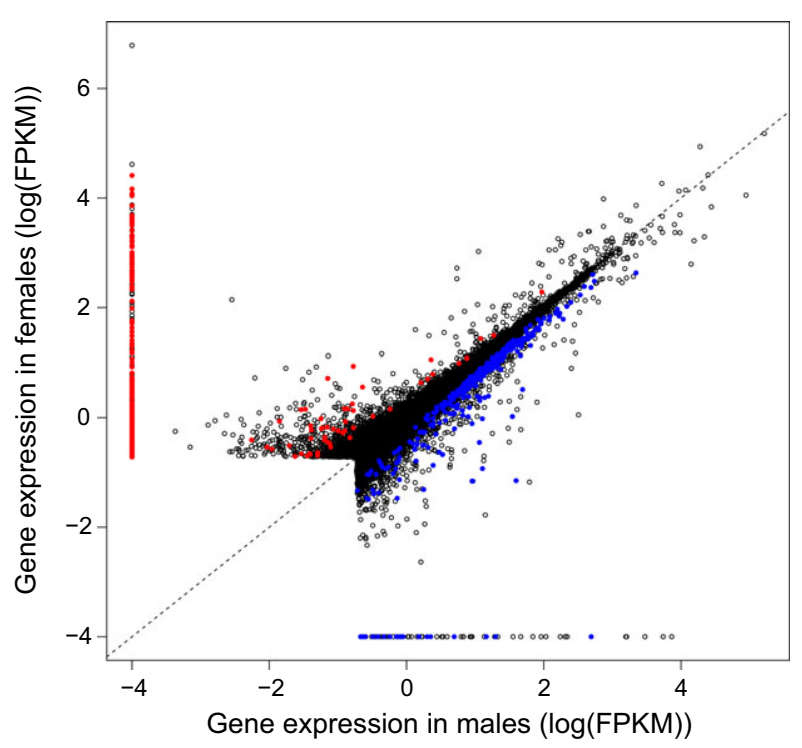

Fig. 3 Sex-specific expression of 24431 genes in the blue tit brain. Genes with low numbers of read alignments in both sexes ( $<10$ fragment alignments) were not tested (lower left corner in plot). The lowest expression in a single sex was set to 0.0001 FPKM $[=-4 \log ($ FPKM $)$, fragments per kilobase of transcript per million mapped reads] and indicates genes with sex-limited expression. Significant male-biased expression is shown in blue and significant female-biased expression in red. The dotted line indicates equal expression. Nonsignificance can also be due to large variance in expression between individuals of one sex.

showed sex-differential expression (1.3\%; Table 1; Fig. 4). Among these, genes with female-biased expression were more common than genes with male-biased expression $\left(\chi^{2}=48.2\right.$, d.f. $\left.=1, P<0.0001\right)$. There is no evidence for enrichment of Z-chromosomal genes within the male-biased gene group in comparison with the female-biased one $\left(\chi^{2}=0.8\right.$, d.f. $\left.=1, P=0.36\right)$. Again, genes that showed sex-biased expression were less likely to be annotated with best protein hits (39\%) than the unbiased genes (75\%). And again, this difference was mainly driven by the low annotation rate among genes with female-biased expression ( $27 \%$ vs. $68 \%$ for male-biased genes).

\section{Characterization of variants}

After filtering, we identified 3964922 heterozygous sites of single nucleotide changes (SNPs) from the genomic sequences in the single male blue tit BT333_1. This translates to one SNP every 277 base pairs. As expected from the general gene size and structure in birds (UCSC browser chicken), $43 \%$ of the polymorphisms were located in genic regions (exons and introns; Table 2). There are more $3^{\prime}$ UTR than $5^{\prime}$ UTR SNPs, as expected because of the larger size of $3^{\prime}$ UTRs. The nonsynonymous SNPs,

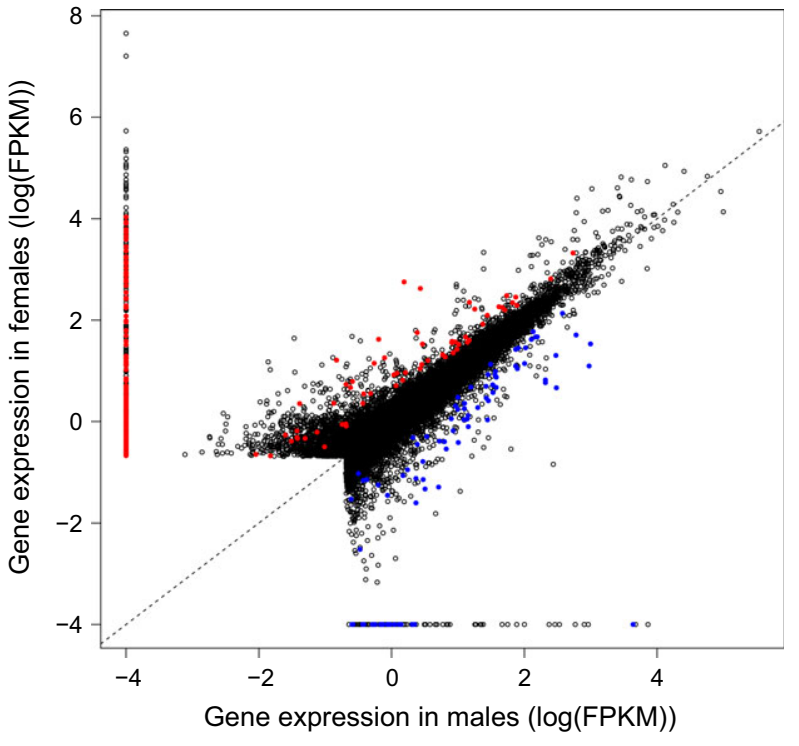

Fig. 4 Sex-specific expression of 22761 genes in the pooled sample of other organs of blue tits. Genes with low numbers of read alignments in both sexes ( $<10$ fragment alignments) were not tested (lower left corner in plot). The lowest expression in a single sex was set to 0.0001 FPKM $(=-4 \log (\mathrm{FPKM})$, fragments per kilobase of transcript per million mapped reads) and indicates genes with sex-limited expression. Significant male-biased expression is shown in blue and significant female-biased expression in red. The dotted line indicates equal expression. Nonsignificance can also be due to large variance in expression between individuals of one sex.

Table 2 Genomic variants determined from DNA-seq data of the reference blue tit according to their location and functional type. Variants can appear in multiple exonic subcategories due to overlapping and nested gene structure. Percentages of the main categories are given in brackets

\begin{tabular}{|c|c|c|}
\hline Variant type & SNPs & Indels \\
\hline Intergenic & $2243928(57 \%)$ & $308270(58 \%)$ \\
\hline Exon & $167551(4 \%)$ & $19251(4 \%)$ \\
\hline Nonsynonymous & 13973 & \\
\hline Synonymous & 44553 & \\
\hline Frameshift & & 693 \\
\hline Nonframeshift & & 386 \\
\hline $5^{\prime}$ UTR & 24184 & 2987 \\
\hline $3^{\prime}$ UTR & 103150 & 15601 \\
\hline Intron (rest) & $1553443(39 \%)$ & $202979(38 \%)$ \\
\hline
\end{tabular}

with potentially strong functional impacts, sum up to 13973 SNPs.

We identified 530500 heterozygous occurrences of insertions or deletions (indels) based on the genomic sequence in the reference bird (Table 2). The distribution across exons, introns and intergenic regions appears to be similar to that of the SNPs, except that the number of 
indels in coding regions is relatively low (only 6\% of exonic indels). This is probably due to selection against indels with strong impact on the protein structure (local codon changes and/or frame shifts).

Based on the transcriptomic sequences, we detected 435143 SNPs in the Cufflinks regions which are common to both sexes. The majority of these SNPs were located in annotated exons and most of these are in the $3^{\prime}$ UTR, in agreement with relatively large $3^{\prime}$ UTRs (Table 3 ). The high proportion of annotated SNPs was also due to the fact that annotated genes were often larger than nonannotated ones. Still, a substantial number of SNPs (70 204) were located distally to known exons, that is in currently nonannotated transcripts. A total of 46741 and 20387 SNPs were detected in the male- and female-specific Cufflinks regions, respectively. Notably, the majority of sexspecific SNPs were located in nonannotated transcribed regions. Also, within the annotated regions, the proportion of nonsynonymous SNPs is relatively high among the sex-specific SNPs (Table 3).

\section{Comparison between genomic and transcriptomic variant calling}

We identified variants (heterozygotes) for the reference male bird (BT333_1) both from DNA and RNA sequences and were thus able to compare the calling success from the two different sources. We identified 235006 SNPs from the DNA sequence data in comparison with 211269 SNPs from the RNA sequence data in the common male Cufflinks regions (187 370 exons across $82.9 \mathrm{Mb}$ ). Eighty per cent of the genomic SNPs were also found in the transcriptomic SNP set and $89 \%$ of the transcriptomic SNPs were validated by genomic SNPs. The called genotypes were identical in $99.99 \%$ of the overlapping SNP set, indicating a high mapping congruence between RNA-seq and DNA-seq data.

We identified 27586 indels in the common male Cufflinks regions from the genomic data and 17913 indels from the transcriptomic data. Forty-one per cent of the genomic indels were also found in the transcriptomic indel set and $63 \%$ of the transcriptomic indels were detected in the genomic set. Indels identified in both sequence sets had identical genotypes in $92 \%$ of the cases. Due to this low cross-validation rate between genomic and transcriptomic indels and the relatively low number of transcriptomic indels in comparison with the SNPs, we excluded indels from further selection analyses.

\section{Selection signatures in the transcriptomic regions}

Mean Tajima's D across all 16,489 informative 25-kb windows (with $>2$ SNPs) was -0.53 , indicating an overall excess of low-frequency variants (Fig. S1, Supporting information). This most likely represents a signal of purifying selection in the transcriptomic regions, but in theory could also be due to population expansion. There was no association of Tajima's $D$ with transcript coverage $(r=-0.01 ; 95 \%$ confidence interval CI: -0.02 to 0.004 ) across $25-\mathrm{kb}$ windows. Only four clusters of strong negative Tajima's $D \quad(2$ adjacent windows with $D<-1.96)$ were identified as potential regions for historical selective sweeps: chr2_9:750 000-775 000 (gene SNRNP48), chr4_6:3 775 000-3 800000 (genes PHKA1, HDAC8), chr14_1:16 775 000-16 800000 (gene KCTD5) and chr21_1:4 625 000-4 650000 (genes SLC35E2B, CDC2L1, MMP23B). This gene list is not enriched for any GO category. No clusters of strong positive Tajima's $D$, indicative of balancing selection, were detected.

Relative extended haplotype homozygosities measured as iHS values are shown across all informative chromosomes and scaffolds (with $>4$ SNPs) in Fig. S2 (Supporting information). There was a weak, but significant, negative association of iHS means with transcript coverage $(r=-0.05 ; 95 \% \mathrm{CI}:-0.06$ to -0.03$)$ across all $25-\mathrm{kb}$ windows, indicative of a weak confounding effect. Based on our definition of clusters of extreme iHS values (see Materials and methods), we identified 15 regions with unusual haplotype structure (Table 4). The most extreme SNPs within these clusters are mostly common 3' UTR SNPs or SNPs from nonannotated regions, except for two common nonsynonymous SNPs in the MKI67 and DNMT3A gene. The haplotype breakdowns of the

\begin{tabular}{lccc}
\hline SNP type & Both sexes & Male-specific & Female-specific \\
\hline Exon & $343649(79 \%)$ & $15576(33 \%)$ & $5002(25 \%)$ \\
$\quad$ Nonsynonymous & 35920 & 3247 & 1020 \\
Synonymous & 91896 & 4565 & 1827 \\
$5^{\prime}$ UTR & 14722 & 3052 & 405 \\
3' UTR & 201111 & 4712 & 1750 \\
Nonannotated & $91494(21 \%)$ & $31165(67 \%)$ & $15385(75 \%)$ \\
Up/downstream (5 kb off exon) & 21290 & 5653 & 3041 \\
Non-up-/downstream & 70204 & 25512 & 12344 \\
\hline
\end{tabular}

Table 3 Transcriptomic SNPs in blue tits according to their location and functional type in the reference protein annotation for the three Cufflinks categories (transcripts expressed in both sexes, expressed in males only and expressed in females only). Percentages of the main categories are given in brackets 
Table 4 Genomic windows with clusters of strong iHS selection signals identified from SNPs in the transcripts common to both sexes. Entries are ordered according to max $|\mathrm{iHS}|$. Although the search for high iHS clusters was performed across all possible 25-kb windows, the size indicated here is the restricted window in which all SNPs with iHS > 3.09 were located

\begin{tabular}{|c|c|c|c|c|c|}
\hline Position & Size (bp) & Genes & $\begin{array}{l}\text { Proportion of } \\
\text { SNPs with } \\
\mid \text { iHS } \mid>3.09\end{array}$ & Max |iHS| (position) & $\begin{array}{l}\text { Frequency of } \\
\text { major allele }\end{array}$ \\
\hline Chr2_2 (3 267 143-3 269 466) & 2323 & - & $12 / 34$ & $6.94(3267266)$ & 0.60 \\
\hline Chr2_5 (11 793 224-11 798 603) & 5379 & FAM135B & $17 / 85$ & -6.41 (11 $798568,3^{\prime}$ UTR) & 0.55 \\
\hline Chr2_1 (18 $761958-18763$ 148) & 1190 & TPK1 & $12 / 27$ & 6.04 (18 $761958,3^{\prime}$ UTR) & 0.60 \\
\hline Chr2_6 (10 958 063-10 961 897) & 3834 & - & $10 / 24$ & $5.94(10961620)$ & 0.60 \\
\hline Chr6_1 (23 $739440-23741$ 191) & 1751 & MKI67 & $11 / 19$ & -5.66 (23 739768, CDS, NS) & 0.65 \\
\hline Chr1_1 (4 124 737-4 128 617) & 3880 & $\begin{array}{l}\text { Hypothetical } \\
\text { protein; micro } \\
\text { RNA cluster }\end{array}$ & $20 / 34$ & -5.42 (4 127 210, intron; flanking) & 0.70 \\
\hline Chr2_3 (4 397 557-4 400 396) & 2839 & C2H8ORF37 & $13 / 34$ & -5.25 (4 $400333,3^{\prime}$ UTR) & 0.55 \\
\hline Chr1_9 (1 000 981-1 002 289) & 1308 & ETNK1 & $14 / 21$ & -5.22 (1 001 189, $3^{\prime}$ UTR $)$ & 0.55 \\
\hline Chr1_4 (19 825 405-19 828 229) & 2824 & PRKX & $14 / 34$ & -5.15 (19 825 991, 3’ UTR) & 0.75 \\
\hline Chr5_1 (24 $999583-25000364)$ & 781 & DNMT3A & $11 / 18$ & -5.01 (25 000298, CDS, NS) & 0.55 \\
\hline Chr14_1 (4 254 439-4 255 759) & 1320 & - & $10 / 21$ & $4.74(4255757)$ & 0.85 \\
\hline Chr2_1 (32 895 288-32 898 683) & 3395 & SLC35B3 & $13 / 38$ & -4.71 (32 895 723, 3’ UTR) & 0.70 \\
\hline Chr1_3 (376 895-378 471) & 1576 & TMEM139 & $10 / 13$ & -4.63 (376 895, 3’ UTR) & 0.50 \\
\hline Chr1_3(26 $867588-26871$ 222) & 3634 & N6AMT1 & $11 / 14$ & 4.54 (26 867588, intron or $3^{\prime}$ UTR) & 0.55 \\
\hline Chr5_1 (14 687 758-14 690 266) & 2508 & - & $13 / 33$ & $-4.30(14689424)$ & 0.70 \\
\hline
\end{tabular}

two alleles at the five SNPs with the highest iHS values are shown in Figs S3-S7 (Supporting information). The major or relatively common minor allele of these SNPs extends in an unusually frequent haplotype (see extended haplotype homozygosity $(\mathrm{EHH})$ plots and bifurcation plots S3-S7). The genes listed in Table 4 are not enriched for any GO category.

We found 2695 common nonsynonymous SNPs with a minor allele frequency of more than 0.35. Some of these common NS-SNPs formed clusters of 7 SNPs within $25 \mathrm{~kb}$ (Table 5). It appears that the clusters show a general enrichment in NS-SNPs (common and noncommon). Interestingly, one of the clusters with a high density of NS-SNPs overlaps with one of the extreme iHS clusters and represents the MKI67 gene (Fig. 1). The genes associated with common NS-SNP clusters are enriched for the GO category 'centrosome localization' $(P=0.027$; genes ASPM and SYNE2). All NS-SNPs within the three clusters of the mentioned genes (MKI67, ASPM, SYNE2) do not show heterozygote excess, are in regions of average genomic coverage and in regions of perfect alignment with known proteins, indicating a high reliability of annotation.

\section{Discussion}

We characterized the genome sequence and transcriptome of a common songbird, the blue tit. The data available via a genome browser - can be used for studies on the genetic architecture and evolutionary potential of ecologically relevant traits. Information and patterns solely based on sequences can be utilized to identify genomic regions of interest, which might then be linked to the evolution of ecologically relevant traits via trait mapping or the analysis of functional pathways. Detecting signals of ongoing selection is of particular interest for micro-evolutionary processes on an ecological timescale (Vitti et al. 2013). An additional approach for the detection of interesting genes in the context of sexual selection and sex-specific behaviour is the analysis of sex-biased expression, because genes that show sex-biased expression are known to evolve rapidly (Parsch \& Ellegren 2013).

\section{Genome and sex-specific transcriptome characterization}

In a first step, we assembled the blue tit genome with high sequence continuity, that is with large superscaffolds (N50 of $16.8 \mathrm{Mb}$ ). This was possible because a high-quality draft genome of the related ground tit was available (Cai et al. 2013; Qu et al. 2013). Similar to the ground tit, a high proportion of the blue tit genome (91\%) could be mapped to all chicken chromosomes except two smaller chromosomes. This is not unexpected given the general high synteny observed among bird species (Ellegren 2013).

In a second step, the transcriptome of five female and five male birds was assembled and mapped to the reference genome. Whereas the majority $(52-75 \%)$ of the predicted genes that expressed at similar levels in both 
Table 5 Genomic windows with clusters of common $(>0.35)$ nonsynonymous SNPS (NS-SNPs) identified from all SNPs in the transcripts common to both sexes. Entries are ordered according to number of common NS-SNPs. Although the search for common NS-SNP clusters was performed across all possible $25-\mathrm{kb}$ windows, the size indicated here is the restricted window in which all common NS-SNPs were located

\begin{tabular}{|c|c|c|c|}
\hline Position & Size $(b p)$ & Genes & $\begin{array}{l}\text { Number of } \\
\text { common } \\
\text { NS-SNPs / } \\
\text { noncommon } \\
\text { NS-SNPs / } \\
\text { S-SNPs }\end{array}$ \\
\hline $\begin{array}{l}\text { Chr3_5 (1 } 020 \text { 415-1 } \\
034 \text { 835) }\end{array}$ & 14420 & GVINP1 & $44 / 124 / 122$ \\
\hline $\begin{array}{l}\text { Scaffold1812_1 } \\
(4334-8810)\end{array}$ & 4476 & LOC101806664 & $30 / 22 / 50$ \\
\hline 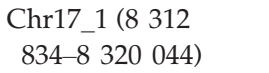 & 7210 & LOC101816715 & $13 / 48 / 21$ \\
\hline $\begin{array}{l}\text { Chr2_4 (11 } 718 \\
\text { 875-11 } 754 \text { 418) }\end{array}$ & 35543 & CEP192 & $11 / 31 / 46$ \\
\hline $\begin{array}{l}\text { Chr6_1 (23 } 729 \\
\text { 867-23 } 739961)\end{array}$ & 10094 & MKI67 & $10 / 42 / 24$ \\
\hline $\begin{array}{l}\text { Chr14_1 (2 } 639 \\
521-2648 \text { 307) }\end{array}$ & 8786 & SLX4 & $9 / 12 / 6$ \\
\hline $\begin{array}{l}\text { Chr8_1 (8 994 } \\
\text { 539-9 } 012440)\end{array}$ & 17901 & ASPM & $9 / 52 / 35$ \\
\hline $\begin{array}{l}\text { Chr1_1 (41 } 521 \\
487-41527470)\end{array}$ & 5983 & DDIAS & $8 / 23 / 16$ \\
\hline $\begin{array}{l}\text { Chr2_1 (22 } 790 \\
125-22800493)\end{array}$ & 10368 & KIAA0947 & $8 / 33 / 11$ \\
\hline $\begin{array}{l}\text { Chr3_2 (8 } 800 \\
604-8821307\end{array}$ & 20703 & MIA3 & $8 / 11 / 24$ \\
\hline $\begin{array}{l}\text { Chr7_3 (290 } \\
\text { 285-294 282) }\end{array}$ & 3997 & LOC101820543 & $8 / 7 / 6$ \\
\hline $\begin{array}{l}\text { Chr1_12 (1 } 793 \\
558-1800896)\end{array}$ & 7338 & $\begin{array}{l}\text { SON or } \\
\text { LOC101920289 }\end{array}$ & $7 / 48 / 57$ \\
\hline Chr11_6 (1873-5970) & 4097 & (HYDIN) & $7 / 2 / 2$ \\
\hline $\begin{array}{l}\text { Chr19_1 (5 } 727 \\
003-5734 \text { 228) }\end{array}$ & 7225 & SPAG5 & $7 / 21 / 13$ \\
\hline $\begin{array}{l}\text { Chr2_3 (2 } 390 \\
\text { 388-2 } 391459)\end{array}$ & 1071 & $\mathrm{FBXO} 43$ & $7 / 1 / 4$ \\
\hline $\begin{array}{l}\text { Chr3_1 (5 467 } \\
\text { 340-5 } 483714)\end{array}$ & 16374 & MDN1 & $7 / 30 / 21$ \\
\hline $\begin{array}{l}\text { Chr5_1 (7 } 033 \\
045-7045444)\end{array}$ & 12399 & SYNE2 & $7 / 16 / 21$ \\
\hline $\begin{array}{l}\text { Chr6_1 (5 727 } 574 \\
-5732587)\end{array}$ & 5013 & CYP2W1 & $7 / 8 / 10$ \\
\hline
\end{tabular}

sexes could be mapped to annotated protein-coding genes, only $39-56 \%$ of the genes showing sex-biased expression were annotated with aligned protein sequences. This could indicate that sex-biased expression often involves noncoding, regulatory elements of the genome, such as long noncoding RNAs (Reinius et al. 2010; Buckberry et al. 2014) or microRNAs (Marco 2014). If this is true, the vast majority of studies on sex-biased gene expression, which only focus on protein-coding genes (Ellegren \& Parsch 2007), may represent only a part of the sex-biased transcriptome. Given that some differentially spliced isoforms might have been missed during the annotation process, it could also mean that some of the sex-biased transcripts represent alternatively spliced exons. Such sex differences in splicing are widespread in the adult human brain (Trabzuni et al. 2013). The regulatory system behind sex-specific expression certainly represents an open field for further studies (Mank et al. 2013).

In contrast to the pooled organ sample which showed only $1.3 \%$ of genes with sex-biased expression, a high proportion of genes expressed in testes and ovaries showed a significant sex bias (53\%). Similarly high percentages of genes with sex-biased expression have been observed in the adult reproductive tissues of other organisms, including chicken (Ellegren \& Parsch 2007; Mank et al. 2010). Whereas there was little difference in the number of genes with male- vs. female-biased expression across all autosomes, male-biased genes were significantly enriched on the Z-chromosome. This finding is consistent with incomplete dosage compensation in the homogametic (ZZ) males (Storchova \& Divina 2006; Parsch \& Ellegren 2013). However, the majority of genes with sex-biased expression appear to be located on the autosomes and hence require a different mechanism for sex-specific expression.

The brain transcriptome also showed a higher proportion of genes with sex-biased expression $(2.5 \%)$ than the transcriptome from the pooled other organs. This proportion is similar to what has been found in the zebra finch and common whitethroat (Sylvia communis) brain (Naurin et al. 2011). Among the 325 genes with malebiased expression, which comprise about half of the genes with sex-biased expression, the majority (63\%) was linked to the Z-chromosome. In contrast, only a small number of genes with female-biased expression was Z-linked (8\%). Interestingly, most of the genes with female-biased expression in the brain were female-limited (expressed exclusively in females), whereas the majority of genes with male-biased expression were expressed in both sexes. This pattern across both autosomes and the Z-chromosome shows some similarity with the expression pattern on the Z-chromosome found in the zebra finch and the common whitethroat brain, where Z-linked male bias in gene expression increased with mean expression level across the sexes (Naurin et al. 2012). Male bias can positively correlate with overall expression level when female-limited genes (i.e. with a negative male bias) are included. We thus hypothesize that the sexual dimorphism in gene expression in the avian brain is based on two distinct components: a qualitative female-limited expression of mostly ncRNA genes and a quantitative male-biased expression of mostly 
coding genes. The male-biased expression is Z-linked and most likely represents chromosomal dosage differences. The exclusive expression of genes in females could provide an explanation for their higher evolutionary rates in comparison with genes with male-biased expression as observed in the chicken brain (Mank et al. 2007).

\section{Selection signals in polymorphism data}

Identifying genomic variants from RNA-seq data is seen as an important add-on to transcriptome assembly and expression analysis, but it also poses a challenge because of the intrinsic complexity in the transcriptome (Piskol et al. 2013; Quinn et al. 2013). We called the RNA-seq variants according to the best practice suggestions of the genome analysis toolkit GATK (Van der Auwera et al. 2013 and associated web pages) and were able to evaluate them with the variants identified from DNA-seq data on the same individual. The cross-validation of our RNA-seq and DNA-seq SNP calling suggests that the reliability was sufficiently high (more than $80 \%$ of SNPs called from one data set were also called in the other data set and the identified genotypes were nearly identical in the overlapping SNP set). The difference in called SNPs from RNA-seq and DNA-seq data can be due to different procedures in mapping, duplicate removal, splice-site alignment and variant filtering, but can also result from differences in read coverage between RNA-seq and DNA-seq data. We assume that allelespecific expression bias is of minor importance in individually called genotypes and does not influence allele frequency estimates. However, the indels identified by RNA-seq and DNA-seq data were quite different. Indel calling is sensitive to local realignment steps, and the guidelines for indel calling from RNA-seq data are still preliminary. We thus disregarded the indels for further analyses on selection signals.

We utilized different polymorphism information to identify selection signals in the genome: allele frequency spectra, EHH and common nonsynonymous coding SNPs. The test statistics (Tajima's $D$ and iHS) appeared to be only weakly confounded by local genomic features such as the density of transcribed regions. However, because iHS is partly based on error-prone long-distance haplotype estimations, we suggest that the identified genomic regions and genes only serve as candidates for further evaluating studies on recent or ongoing adaptive processes in blue tit populations. The gene lists obtained through the first two approaches were not enriched for any specific functional annotation compared to the complete gene composition. However, the list of genes with clusters of common nonsynonymous SNPs was functionally enriched for the term 'centrosome localization'. The products of the identified genes ASPM and SYNE2 play important roles in the establishment and maintenance of centrosome localization in the cytosol during mitosis and meiosis. The dynamic evolution of meiotic drive of selfish gene elements predicts rapid evolution of proteins associated with chromosome movements during meiosis (Henikoff et al. 2001; Axelsson et al. 2010). The enrichment of common nonsynonymous SNPs could thus indicate an ongoing or partial selective sweep of structural variants in genes associated with chromosome separation, for which centrosome positioning plays a role. The observation of incomplete selective sweeps is not unlikely if repeated selective events related to the evolution of meiotic drive are predicted. However, it could also indicate balancing selection among different structural variants of the genes. For the ASPM gene, which shows positive selection during primate evolution (Mekel-Bobrov et al. 2005), functions like the regulation of brain and gonad development have been reported (Pulvers et al. 2010).

One gene (MKI67) was identified in two different approaches and showed clusters of extreme $\mathrm{EHH}$ at common nonsynonymous SNPs. It is therefore a good candidate for a partial selective sweep on structural variants. The gene product of MKI67 is a well-known marker for cell proliferation in cancer medicine, but little is known about its molecular function (Scholzen \& Gerdes 2000). Because of its centromere localization during specific stages in the mitotic and meiotic cell cycle (Traut et al. 2002), it might also have an important function during meiotic chromosome movements, similar to ASPM and SYNE2. It therefore represents another candidate for repeated meiotic drive evolution.

\section{Acknowledgements}

We thank Lisa Trost, Agnes Tuerk, Antje Girndt, Silke Laucht, Kim Teltscher and Christine Baumgartner for help in the field and laboratory, and the editor Anna Santure and three anonymous reviewers for helpful comments on the manuscript. This work was funded by the Max Planck Society.

\section{References}

Arct A, Drobniak SM, Podmokła E, Gustafson L, Cichoń M (2013) Benefits of extra-pair mating may depend on environmental conditions an experimental study in the blue tit (Cyanistes caeruleus). Behavioral Ecology and Sociobiology, 67, 1809-1815.

Axelsson E, Albrechtsen A, van AP et al. (2010) Segregation distortion in chicken and the evolutionary consequences of female meiotic drive in birds. Heredity, 105, 290-298.

Buckberry S, Bianco-Miotto T, Bent SJ, Dekker GA, Roberts CT (2014) Integrative transcriptome metaanalysis reveals widespread sex-biased gene expression at the human fetal-maternal interface. Molecular Human Reproduction, 20, 810-819.

Cai Q, Qian X, Lang Y et al. (2013) Genome sequence of ground tit Pseudopodoces humilis and its adaptation to high altitude. Genome Biology, 14, R29. 
Cingolani P, Platts A, Wang LL et al. (2012) A program for annotating and predicting the effects of single nucleotide polymorphisms, SnpEff: SNPs in the genome of Drosophila melanogaster strain w1118; iso-2; iso3. Fly, 6, 1-13.

Clutton-Brock TH, Sheldon BC (2010) Individuals and populations: the role of long-term, individual-based studies in ecology and evolutionary biology. Trends in Ecology and Evolution, 25, 562-573.

Dalloul RA, Long JA, Zimin AV et al. (2010) Multi-platform next-generation sequencing of the domestic turkey (Meleagris gallopavo): genome assembly and analysis. PLoS Biology, 8, e1000475.

Danecek P, Auton A, Abecasis G et al. (2011) The variant call format and VCFtools. Bioinformatics, 27:2156-2158.

Delaneau O, Howie B, Cox A, Zagury JF, Marchini J (2013) Haplotype estimation using sequence reads. American Journal of Human Genetics, 93, 787-796.

DePristo M, Banks E, Poplin R et al. (2011) A framework for variation discovery and genotyping using next-generation DNA sequencing data. Nature Genetics, 43, 491-498.

Diz AP, Martinez-Fernandez M, Rolan-Alvarez E (2012) Proteomics in evolutionary ecology: linking the genotype with the phenotype. Molecular Ecology, 21, 1060-1080.

Ellegren H (2010) Evolutionary stasis: the stable chromosomes of birds. TREE, 25, 283-291.

Ellegren H (2013) The evolutionary genomics of birds. Annual Review of Ecology and Systematics, 44, 239-259.

Ellegren H, Parsch J (2007) The evolution of sex-biased genes and sex-biased gene expression. Nature Reviews Genetics, 8, 689-698.

Ellegren H, Sheldon BC (2008) Genetic basis of fitness differences in wild populations. Nature, 452, 169-175.

Ellegren H, Smeds L, Burri R et al. (2012) The genomic landscape of species divergence in Ficedula flycatchers. Nature, 491, 756-760.

Frankl-Vilches C, Kuhl H, Werber M et al. (2015) Using the canary genome to decipher the evolution of hormone-sensitive gene regulation in seasonal singing birds. Genome Biology, 16, 19. doi:10.1186/s13059-0140578-9.

Gautier M, Vitalis R (2012) rehh: an R package to detect footprints of selection in genome-wide SNP data from haplotype structure. Bioinformatics, 28, 1176-1177.

Gotoh O (2008) A space-efficient and accurate method for mapping and aligning cDNA sequences onto genomic sequence. Nucleic Acids Research, 36, 2630-2638.

Guttman M, Amit I, Garber M et al. (2009) Chromatin signature reveals over a thousand highly conserved large non-coding RNAs in mammals. Nature, 12, 223-227.

Henikoff S, Ahmad K, Malik HS (2001) The centromere paradox: stable inheritance with rapidly evolving DNA. Science, 293, 1098-1102.

Huang Y, Li Y, Burt DW et al. (2013) The duck genome and transcriptome provide insight into an avian influenza virus reservoir species. Nature Genetics, 45, 776-783.

International Chicken Genome Sequencing Consortium (ICGSC) (2004) Sequence and comparative analysis of the chicken genome provide unique perspectives on vertebrate evolution. Nature, 432, 695-716.

Kielbasa SM, Wan R, Sato K, Horton P, Frith MC (2011) Adaptive seeds tame genomic sequence comparison. Genome Research, 21, 487.

Kim D, Pertea G, Trapnell C, Pimentel H, Kelley R, Salzberg SL (2011) TopHat2: accurate alignment of transcriptomes in the presence of insertions, deletions and gene fusions. Genome Biology, 14, R36.

Kluen E, Kuhn S, Kempenaers B, Brommer JE (2012) A simple cage test captures intrinsic differences in aspects of personality across individuals in a passerine bird. Animal Behaviour, 84, 279-287.

Kluyver HN (1951) The population ecology of the Great Tit, Parus $m$. major L. Ardea, 39, 1-135.

Lack D (1964) A long-term study of the great tit (Parus major). Journal of Animal Ecology, 33, 159-173.

Mank JE, Hultin-Rosenberg L, Axelsson E, Ellegren H (2007) Rapid evolution of female-biased, but not male-biased, genes expressed in the avian brain. Molecular Biology and Evolution, 24, 2698-2706.
Mank JE, Nam K, Brunstroem B, Ellegren H (2010) Ontogenetic complexity of sexual dimorphism and sex-specific selection. Molecular Biology and Evolution, 27, 1570-1578.

Mank JE, Wedell N, Hosken DJ (2013) Polyandry and sex-specific gene expression. Philosophical Transactions of the Royal Society B: Biological Sciences, 368, 20120047

Marco A (2014) Sex-biased expression of microRNAs in Drosophila melanogaster. Open Biology, 4, 140024.

Mekel-Bobrov N, Gilbert SL, Evans PD et al. (2005) Ongoing adaptive evolution of ASPM, a brain size determinant in Homo sapiens. Science, 309, 1720-1722.

Merilä J, Sheldon BC (1999) Genetic architecture of fitness and nonfitness traits: empirical patterns and development of ideas. Heredity, 83, 103109

Myers EW, Sutton GG, Delcher AL et al. (2000) A whole-genome assembly of Drosophila. Science, 287, 2196-2204.

Naurin S, Hansson B, Hasselquist D, Kim Y-H, Bensch S (2011) The sexbiased brain: sexual dimorphism in gene expression in two species of songbirds. BMC Genomics, 12, 37.

Naurin S, Hasselquist D, Bensch S, Hansson B (2012) Sex-biased gene expression on the avian $\mathrm{Z}$ chromosome: highly expressed genes show higher male-biased expression. PLoS One, 7, e46854.

Necsulea A, Kaessmann H (2014) Evolutionary dynamics of coding and non-coding transcriptomes. Nature Review Genetics, 15, 734-748.

Olano-Marin J, Dawson DA, Girg A et al. (2010) A genome-wide set of 106 microsatellite markers for the blue tit (Cyanistes caeruleus). Molecular Ecology Resources, 10, 516-532.

Olano-Marin J, Mueller JC, Kempenaers B (2011a) Heterozygosity and survival in blue tits (Cyanistes caeruleus): contrasting effects of presumably functional and neutral loci. Molecular Ecology, 20, 40284041.

Olano-Marin J, Mueller JC, Kempenaers B (2011b) Correlations between heterozygosity and reproductive success in the blue tit (Cyanistes caeruleus): an analysis of inbreeding and single locus effects. Evolution, 65, 3175-3194.

Parsch J, Ellegren H (2013) The evolutionary causes and consequences of sex-biased gene expression. Nature Reviews Genetics, 14, 83-87.

Peng Y, Leung HC, Yiu SM, Chin FY (2012) IDBA-UD: a de novo assembler for single-cell and metagenomic sequencing data with highly uneven depth. Bioinformatics, 28, 1420-1428.

Philippe N, Samra EB, Boureux A et al. (2014) Combining DGE and RNA-sequencing data to identify new polyA+ non-coding transcripts in the human genome. Nucleic Acids Research, 42, 2820-2832.

Piskol R, Ramaswami G, Li JB (2013) Reliable identification of genomic variants from RNA-seq data. American Journal of Human Genetics, 93, 641-651.

Poelstra JW, Vijay N, Bossu CM et al. (2014) The genomic landscape underlying phenotypic integrity in the face of gene flow in crows. Science, 344, 1410-1414.

Pulvers JN, Bryk J, Fish JL et al. (2010) Mutations in mouse Aspm (abnormal spindle-like microcephaly associated) cause not only microcephaly but also major defects in the germline. Proceedings of the National Academy of Sciences of the United States of America, 107, 16595-16600.

Qu Y, Zhao H, Han N et al. (2013) Ground tit genome reveals avian adaptation to living at high altitudes in the Tibetan plateau. Nature Communications, 4, 2071

Quinlan AR, Hall IM (2010) BEDTools: a flexible suite of utilities for comparing genomic features. Bioinformatics, 26, 841-842.

Quinn EM, Cormican P, Kenny EM et al. (2013) Development of strategies for SNP detection in RNA-seq data: application to lymphoblastoid cell lines and evaluation using 1000 Genomes data. PLoS One, 8, e58815.

Reimand J, Arak T, Vilo J (2011) g:Profiler - a web server for functional interpretation of gene lists (2011 update). Nucleic Acids Research, 39, W307-W315.

Reinius B, Shi C, Hengshuo L et al. (2010) Female-biased expression of long non-coding RNAs in domains that escape X-inactivation in mouse. BMC Genomics, 11, 614 
Schlicht E, Kempenaers B (2013) Effects of social and extra-pair mating on sexual selection in blue tits (Cyanistes caeruleus). Evolution, 67, 14201434.

Scholzen T, Gerdes J (2000) The Ki-67 protein: from the known and the unknown. Journal of Cellular Physiology, 182, 311-322.

Seutin G, White BN, Boag PT (1991) Preservation of avian blood and tissue samples for DNA analysis. Canadian Journal of Zoology, 69, 82-90.

Shapiro MD, Kronenberg Z, Li C et al. (2013) Genomic diversity and evolution of the head crest in the rock pigeon. Science, 339, 1063-1067.

Springer SA, Crespi BJ, Swanson WJ (2011) Beyond the phenotypic gambit: molecular behavioural ecology and the evolution of genetic architecture. Molecular Ecology, 20, 2240-2257.

Steinmeyer C, Kempenaers B, Mueller JC (2012) Testing for associations between candidate genes for circadian rhythms and individual variation in sleep behaviour in blue tits. Genetica, 140, 219-228.

Storchova R, Divina P (2006) Nonrandom representation of sex-biased genes on chicken $\mathrm{Z}$ chromosome. Journal of Molecular Evolution, 63, 676-681.

Trabzuni D, Ramasamy A, Imran S et al. (2013) Widespread sex differences in gene expression and splicing in the adult human brain. Nature Communications, 4, 2771.

Trapnell C, Williams BA, Pertea G et al. (2010) Transcript assembly and quantification by RNA-Seq reveals unannotated transcripts and isoform switching during cell differentiation. Nature Biotechnology, 28, 511-515.

Trapnell C, Hendrickson DG, Sauvageau M, Goff L, Rinn JL, Pachter L (2013) Differential analysis of gene regulation at transcript resolution with RNA-seq. Nature Biotechnology, 31, 46-53.

Traut W, Endl E, Scholzen T, Gerdes J, Winking H (2002) The temporal and spatial distribution of the proliferation associated Ki-67 protein during female and male meiosis. Chromosoma, 111, 156-164.

Van der Auwera GA, Carneiro M, Hartl C et al. (2013) From FastQ data to high-confidence variant calls: the genome analysis toolkit best practices pipeline. Current Protocols in Bioinformatics, 43, 11.10.1-11.10.33.

Van Oers K, Mueller JC (2010) Evolutionary genomics of animal personality. Philosophical Transactions of the Royal Society B: Biological Sciences, 365, 3991-4000.

Vedder O, Komdeur J, van der Velde M, Schut E, Magrath MJL (2011) Polygyny and extra-pair paternity enhance the opportunity for sexual selection in blue tits. Behavioral Ecology and Sociobiology, 65, $741-752$.

Vitti JJ, Grossman SR, Sabeti PC (2013) Detecting natural selection in genomic data. Annual Review of Genetics, 47, 97-120.

Voight BF, Kudaravalli S, Wen X, Pritchard JK (2006) A map of recent positive selection in the human genome. PLoS Biology, 4, e72.

Warren WC, Clayton DF, Ellegren $\mathrm{H}$ et al. (2010) The genome of a songbird. Nature, 464, 757-762.

Zhan X, Pan S, Wang J et al. (2013) Peregrine and saker falcon genome sequences provide insights into evolution of a predatory lifestyle. $\mathrm{Na}$ ture Genetics, 45, 563-568.

Zhang G, Li C, Li Q et al. (2014) Comparative genomics reveals insights into avian genome evolution and adaptation. Science, 346, 1311-1320.

J.C.M. conceived of the study, participated in the study design and coordination, performed the differential expression, selection and statistical analyses and drafted the manuscript. H.K. participated in the study design and coordination, assembled and annotated the genome, mapped the transcriptome and helped draft the manuscript. B.T. participated in the study design and coordination. B.K. conceived of the study, participated in the study design and coordination and revised the manuscript. All authors read and approved the final manuscript.

\section{Data Accessibility}

The blue tit genome browser is available at http://public-genomes-ngs.molgen.mpg.de/. The raw sequences are deposited at the NCBI/SRA archive under bioproject no. PRJNA284903. Individual accession numbers are listed in Table S1. Files of annotated SNPs, sex-differential expression results and selection scans can be found under Dryad doi:10.5061/dryad.dc56b.

\section{Supporting Information}

Additional Supporting Information may be found in the online version of this article:

Appendix S1 Command lines with parameters used for assembly, mapping and variant calling/selection.

Fig. S1 Histogram of all Tajima's $D$ values.

Fig. S2 Absolute iHS values across all informative chromosomes/scaffolds (different colours; only larger chromosomes named).

Fig. S3 (A) Breakdown of EHH over physical distance for both allels of the focal SNP chr2_2:3 267266 (dashed line). (B) Bifurcation plots showing the haplotype-breakdown on both sides of the focal SNP: major allele $(60 \%)$ in blue, minor allele $(40 \%)$ in red.

Fig. S4 (A) Breakdown of EHH over physical distance for both allels of the focal SNP chr2_5:11 798568 in gene FAM135B (dashed line). (B) Bifurcation plots showing the haplotypebreakdown on both sides of the focal SNP: major allele $(55 \%)$ in blue, minor allele $(45 \%)$ in red.

Fig. S5 (A) Breakdown of EHH over physical distance for both allels of the focal SNP chr2_1:18 761958 in gene TPK1 (dashed line). (B) Bifurcation plots showing the haplotype-breakdown on both sides of the focal SNP: major allele $(60 \%)$ in blue, minor allele (40\%) in red.

Fig. S6 (A) Breakdown of EHH over physical distance for both allels of the focal SNP chr2_6:10 961620 (dashed line). (B) Bifurcation plots showing the haplotype-breakdown on both sides of the focal SNP: major allele $(60 \%)$ in blue, minor allele $(40 \%)$ in red.

Fig. S7 (A) Breakdown of EHH over physical distance for both allels of the focal SNP chr6_1:23 739768 in gene MKI67 (dashed line). (B) Bifurcation plots showing the haplotype-breakdown on both sides of the focal SNP: major allele $(65 \%)$ in blue, minor allele (35\%) in red.

Table S1 Sequencing details, barcodes and accession numbers.

Table S2 Assignment of blue tit superscaffolds to zebra finch chromosomes. 